\title{
(6) OPEN ACCESS \\ EVEREST study report 2: imaging and grading protocol, and baseline characteristics of a randomised controlled trial of polypoidal choroidal vasculopathy
}

\author{
Colin S Tan, ${ }^{1,2}$ Wei Kiong Ngo, ${ }^{2}$ Jian Ping Chen, ${ }^{2}$ Nikolle W Tan, ${ }^{1,2}$ Tock Han Lim, ${ }^{1,2}$ \\ on behalf of the EVEREST Study Group
}

\begin{abstract}
- Additional material is published online only. To view please visit the journal online (http://dx.doi.org/10.1136/ bjophthalmol-2014-305674).

${ }^{1}$ Fundus Image Reading Centre, National Healthcare Group Eye Institute, Singapore, Singapore

${ }^{2}$ National Healthcare Group Eye Institute, Tan Tock Seng Hospital, Singapore, Singapore
\end{abstract}

\section{Correspondence to} A/Prof Tock Han Lim, National Healthcare Group Eye Institute, Tan Tock Seng Hospital, 11 Jalan Tan Tock Seng, Singapore 308433, Singapore; Tock_Han_Lim@nhg.com.sg

Received 13 June 2014 Accepted 25 September 2014 Published Online First 10 March 2015

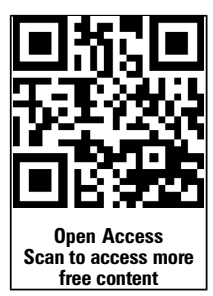

\section{CrossMark}

To cite: Tan CS, Ngo WK,

Chen JP, et al. Br J

Ophthalmol 2015;99:

624-628.

\section{ABSTRACT}

Purpose To describe the imaging standards, grading protocol and baseline characteristics of polypoidal choroidal vasculopathy (PCV) from the EVEREST study. Methods In a prospective, multicentre study, confocal scanning laser ophthalmoscope indocyanine green angiography (ICGA) was performed using a standardised imaging protocol. All images were graded using standardised, calibrated equipment by fellowship-trained ophthalmologists at the Central Reading Center. Results Sixty-one patients with PCV were included in the study. ICGA characteristics included: nodular appearance stereoscopically (56 eyes, 91.8\%), hypofluorescent halo $(42,68.9 \%)$, abnormal vascular network (54, 88.5\%) and pulsation of the polyps $(4,6.6 \%)$. Colour fundus photography revealed orange subretinal nodules $(34,55.7 \%)$ and massive submacular haemorrhage $(8,13.1 \%)$. The mean area of the PCV lesion was $3.11 \mathrm{~mm}^{2}$ (range, $0.2-10.7 \mathrm{~mm}^{2}$ ). The vascular channels filled within 7.3-32.0 s (mean: $17.9 \mathrm{~s}$ ) while the mean filling time for polyps was $21.9 \mathrm{~s}$ (range, 7.3-40.4 s). Patients with massive submacular haemorrhage were less likely to have abnormal vascular channels seen on ICGA (28.6\% vs $83.3 \%$ for those without massive haemorrhage, $p=0.001$ ).

Conclusions The imaging and grading protocols and baseline characteristics of a multicentre, randomised controlled trial of PCV are described in detail, and may serve as reference for future randomised, controlled trials on PCV.

Clinical trial number This work was supported by Novartis Pharma AG, Basel, Switzerland grant number NCT00674323 (clinicaltrials.gov).

\section{INTRODUCTION}

Polypoidal choroidal vasculopathy (PCV) manifests with a variety of clinical presentations, such as serous and/or haemorrhagic detachments of the retinal pigment epithelium (RPE), serous subretinal fluid mimicking central serous chorioretinopathy, or as quiescent orange-red subretinal nodules. ${ }^{1-5}$ In many cases, PCV is clinically indistinguishable from occult choroidal neovascularisation due to neovascular age-related macular degeneration (AMD) and indocyanine green angiography (ICGA) is required to distinguish the two conditions. ${ }^{467}$

The differentiation of PCV from AMD is clinically relevant because the treatment responses of two diseases differ. ${ }^{8}{ }^{9}$ While AMD has been shown to respond well to antivascular endothelial growth factor monotherapy, studies such as the EVEREST study ${ }^{10}$ have demonstrated better rates of polyp closure and improvements in visual acuity among patients receiving combination therapy with photodynamic therapy and intravitreal ranibizumab. ${ }^{9}$ In addition, there are reports of patients with presumed AMD who responded poorly to prolonged treatment with antivascular endothelial growth factor monotherapy. ICGA performed subsequently confirmed the diagnosis of PCV, and treatment with photodynamic therapy led to resolution of the lesions with improvements in visual acuity. ${ }^{9}$

The accurate diagnosis of PCV is essential to ensure appropriate treatment and to allow comparison among various studies on PCV treatment. Although the ICGA features of PCV have been described to various extents in the literature, ${ }^{4} 7811-14$ there are significant differences in the interpretation of ICGA for the diagnosis of PCV. In addition, several clinical conditions may mimic the appearance of PCV on ICGA, hence the need to use consistent diagnostic features of PCV.

The imaging methods and diagnostic criteria were only briefly discussed in the first report of the EVEREST Study. ${ }^{10}$ This paper aims to provide further details and explain the rationale of the imaging standards and grading protocol, and to describe the baseline ICGA lesion characteristics of the patients.

\section{METHODS}

This study was approved by the respective institutional review boards of the study sites. Written, informed consent was obtained from all patients prior to enrolment in the study.

\section{Imaging protocol}

Fluorescein angiograms and ICGA were performed using a confocal scanning laser ophthalmoscope (Heidelberg Retinal Angiograph (HRA), HRA-C/ HRA2/HRA Spectralis, Heidelberg Engineering, Germany). When performing confocal scanning laser ophthalmoscope angiograms, the dose of indocyanine green (ICG) required is significantly lower compared with flash ICGA. ICG $10 \mathrm{mg}$ in $2 \mathrm{~mL}$ using the accompanying aqueous diluent kit (Akorn, Pulsion or equivalent, as approved by the local health authority) was injected through the anterior cubital fossa vein over $\leq 5 \mathrm{~s}$.

The standard angiographic field for all frames was $30^{\circ}$ centred on the fovea, with a resolution of 
$1536 \times 1536$ pixels and capturing speed of $\geq 6$ frames per second for dynamic angiography. For still-frames, image quality was improved by digital signal averaging using either the automated real-time function or the 'mean' function.

The timer was started at the start of the bolus injection, while the capture button was triggered only at first dye appearance. The 'precapture' mode of the HRA device allows recording of $2 \mathrm{~s}$ of the angiogram prior to the triggering of the capturing button, which is very useful for capturing the initial frames when the dye just enters the choroidal circulation through the feeder vessel. In order to image the full filling sequence of the branching vascular network (BVN), the dynamic capture duration was set at $\geq 30 \mathrm{~s}$.

Still-frame captures were performed according to the sequence as shown in online supplementary appendix 1. Stereo-pair images were obtained during each time period. To obtain stereo-pair images, one frame was taken (using the automated real-time function) and the device was shifted laterally within the same plane, slightly to the right, and the second stereo-pair image taken.

\section{Case definition of PCV}

The Central Reading Center used standardised criteria to diagnose $\mathrm{PCV}^{4}{ }^{7}$ the features of which were distilled from available English literature from 1990 to 2008. PCV was diagnosed based on early subretinal ICGA hyperfluorescence (appearing within the first $5 \mathrm{~min}$ of ICG dye injection) and at least one of the following diagnostic criteria (figure 1 ):

- Nodular appearance of the polyp on stereoscopic viewing

- Hypofluorescent halo around the nodule

- Abnormal vascular channel(s) supplying the polyps

- Pulsatile filling of polyps

- Orange subretinal nodules corresponding to the hyperfluorescent area on ICGA

- Massive submacular haemorrhage.

Two types of abnormal vascular channels were identified using dynamic ICGA (figure 2). ${ }^{7}$ A typical BVN originates from a feeder vessel and spreads outwards, with polyps typically located at the periphery. A second, less common type was interconnecting channels, which consisted of a network of crisscrossing choroidal vessels which supply the associated polyps. The dye has no specific point of origin or direction of flow, unlike a BVN.

\section{Grading protocol}

All images were graded by trained graders (Fellowship-trained retinal specialists) at the Fundus Image Reading Center, National Healthcare Group Eye Institute, Singapore, using standardised equipment and grading protocols. High resolution $(3000 \times 2000)$ monitors (HP LP3065, Hewlett Packard, Palo Alto, California, USA) were used to view all images for stereoscopic viewing at the full resolution of the ICGA image. The monitors were calibrated weekly using the Spyder 3 calibration software (Datacolor, Lawrenceville, New Jersey, USA).

ICGA images were graded using the Heidelberg Eye Explorer software (Heidelberg Engineering, Heidelberg, Germany). The dynamic ICGA was played for the entire $30 \mathrm{~s}$. The slider on the viewing software was then moved to the point when the ICG dye first appeared and advanced slowly to allow visualisation of the initial filling of the feeder vessels and polyps. In addition, the pulsation of the polyps was determined using the dynamic ICGA.
The filling times were read from the timer of the viewing software. Vascular channel filling time was defined as the first time the ICG dye was visible within the abnormal vascular channels. Polyp filling time was defined as the time of first dye appearance within a polyp.

A best-fit circle was drawn around each polyp using the drawing tools on the Heidelberg Eye Explorer software (figure $3 \mathrm{~A}$ ). The diameter of each circle was used to calculate the area of each polyp. The total lesion area was determined using the freehand drawing tool. The grader drew an outline which encompassed all polyps identified and the area of the abnormal vascular channels (identified using dynamic ICGA), and the software returned the total area in $\mathrm{mm}^{2}$. The circle drawing tool was then used to draw a best-fit circle around the total lesion area, the diameter of which was the greatest linear dimension (figure 3B).

Stereo-pair colour fundus photographs were viewed using the Visupac viewer V.4.4.4 (Carl Zeiss Meditec, Dublic, California, USA) and the Screen-Vu stereoscope. The presence of orange subretinal nodules and massive submacular haemorrhage (defined as submacular haemorrhage $\geq 4$ disc areas) was noted from the colour fundus photographs.

\section{RESULTS}

Of 95 cases submitted for screening, 61 were diagnosed with PCV. The mean age was 65 years (range, 46-84 years, SD \pm 9.2 years), with 41 men $(67.2 \%)$ and 20 women $(32.8 \%)$. All patients were of Asian ethnicity. In 32 patients, the right eye was involved (53.5\%) whereas the left eye was involved in 29 patients $(47.5 \%)$.

The frequency of ICGA characteristics were as follows: nodular appearance, 56 (91.8\%); hypofluorescent halo around the nodule, 42 (68.9\%); presence of abnormal vascular network, 54 (88.5\%); and pulsation of the polyp(s) on dynamic ICGA, 4 (6.6\%). Using colour fundus photography, 34 (55.7\%) had orange subretinal nodules and $8(13.1 \%)$ presented with massive submacular haemorrhage.

The characteristics of the polyps and BVN are summarised in table 1 . The number of polyps ranged from 1 to 9 , with a median of 4 . The polyp configurations included solitary-16 (26.2\%), ring-28 (45.9\%), cluster-15 (24.6\%) and mixed configurations-2 (3.3\%). The mean total polyp area was $0.25 \mathrm{~mm}^{2}$ (median $0.21 \mathrm{~mm}^{2}$; range, $0.025-2.11 \mathrm{~mm}^{2}$, SD $\pm 0.28 \mathrm{~mm}^{2}$ ). The area of the PCV lesion (comprising polyps and associated vascular channels) ranged from $0.2 \mathrm{~mm}^{2}$ to $10.7 \mathrm{~mm}^{2}$, with a mean of $3.11 \mathrm{~mm}^{2}$ and a median of $2.28 \mathrm{~mm}^{2}$.

Of the cases with associated vascular channels, 46 (75.4\%) had a distinct BVN, whereas $8(13.1 \%)$ were classified as interconnecting channels, as described above. The vascular channels (either BVN or interconnecting channels) filled within 7.3-32.0 s, with a mean of $17.9 \mathrm{~s}$ (median: $17.6 \mathrm{~s}$ ) while the mean filling time for the polyps was $21.9 \mathrm{~s}$ (median: $22.0 \mathrm{~s}$; range, $7.3-40.4 \mathrm{~s}, \mathrm{SD} \pm 8.1 \mathrm{~s}$ ).

The patient manifested with a variety of clinical presentations, including serous subretinal fluid (28 (45.9\%)), serosanguinous (23 (37.7\%)), haemorrhagic (2 (3.3\%)) and massive submacular haemorrhage (8 (13.1\%)). Patients with massive submacular haemorrhage were less likely to have abnormal vascular channels on ICGA $37.5 \%$ vs $83.0 \%$ for those without massive haemorrhage, $p=0.001)$. A higher percentage of patients with pulsation of the polyp on dynamic ICGA presented with haemorrhage of any size, 1 of $4(25.0 \%)$ compared with 9 of 57 $(15.8 \%)$ for those without pulsation, but the difference was not statistically significant $(\mathrm{p}=0.63)$. The frequency of 
Figure 1 Standardised diagnostic features of polypoidal choroidal vasculopathy seen on indocyanine green angiography (ICGA) and colour fundus photography. (A) Colour fundus photograph showing orange subretinal nodules (arrow) and pigment epithelial detachment (arrowhead). (B) ICGA of the same eye demonstrating nodular areas of hyperfluorescence (arrow), appearing within the first $5 \mathrm{~min}$, with a halo of hypofluorescence around the nodule (arrowhead). A branching vascular network (BVN) is seen superior to the fovea, supplying the polyps which are located at the periphery of the BVN. (C) Colour fundus photograph showing massive submacular haemorrhage at the posterior pole. (D) Corresponding ICGA demonstrating areas of

hyperfluorescence (arrow) which correspond with the polyps. No BVN is seen in this angiogram.
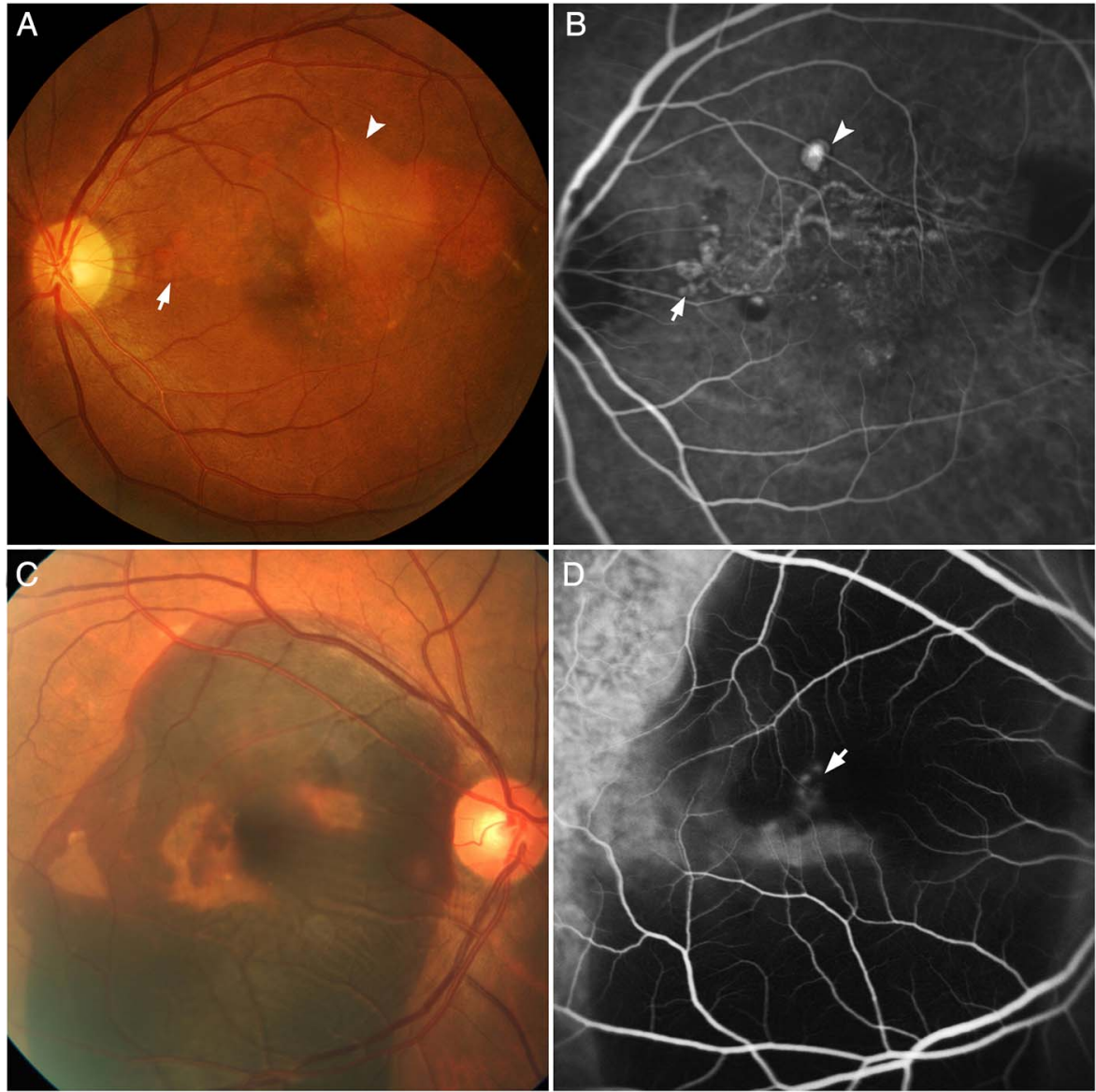

haemorrhagic presentations did not differ between those with orange subretinal nodules (5 patients, $14.7 \%$ ) and those without nodules (5 patients, $18.5 \%$ ).

\section{DISCUSSION}

In this paper, we have described the ICGA imaging standards, grading methods and diagnostic criteria used in the EVEREST Study, ${ }^{10}$ the first multicentre randomised controlled trial of PCV treatment. The baseline ICGA and colour fundus photograph characteristics of patients with PCV are also described. These methods may provide the reference for standardised imaging and grading protocols for PCV in future multicentre studies.
The relevance of the diagnostic criteria can be seen from the results of this paper. While earlier studies on PCV used a variety of clinical and ICGA features to diagnose PCV and differentiate it from neovascular AMD, these features were highly variable between studies and the characteristic appearance of a polyp was often not well-defined. In many papers, the polyps have been described as 'polypoidal choroidal vascular dilatations', 15 'focal vascular dilations'6 $12-141617$ or 'polypoidal lesions'. ${ }^{18}$ These terms, however, may mean different things and be interpreted differently by various clinicians. Therefore, it is imperative to use standardised criteria that are easy to apply across different studies in order to ensure that the results of these studies are comparable. In this study, three parameters were
Figure 2 Indocyanine green angiograms (ICGA) of two cases of polypoidal choroidal vasculopathy, illustrating the types of abnormal vascular channels. (A) ICGA showing interconnecting channels superotemporal to the fovea (oval outline). (B) ICGA demonstrating a characteristic branching vascular network centrally (arrows), with polyps superior and inferior to the network.
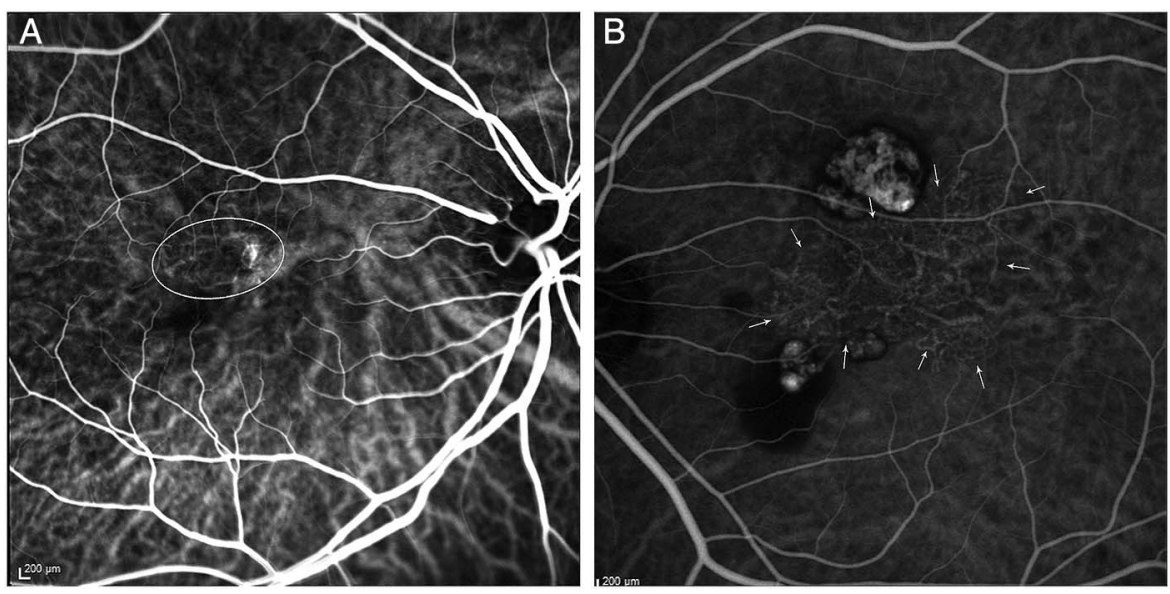
Figure 3 Grading diagrams from the Central Reading Center. (A) Grading diagram illustrating the location of each polyp, with the diameter of each polyp shown by the figures in green. (B) Grading diagram illustrating the outline of the entire polypoidal choroidal vasculopathy (PCV) lesion (green), with a best-fit circle (yellow) used to calculate the greatest linear dimension (GLD). The area of the PCV lesion and the GLD are shown.
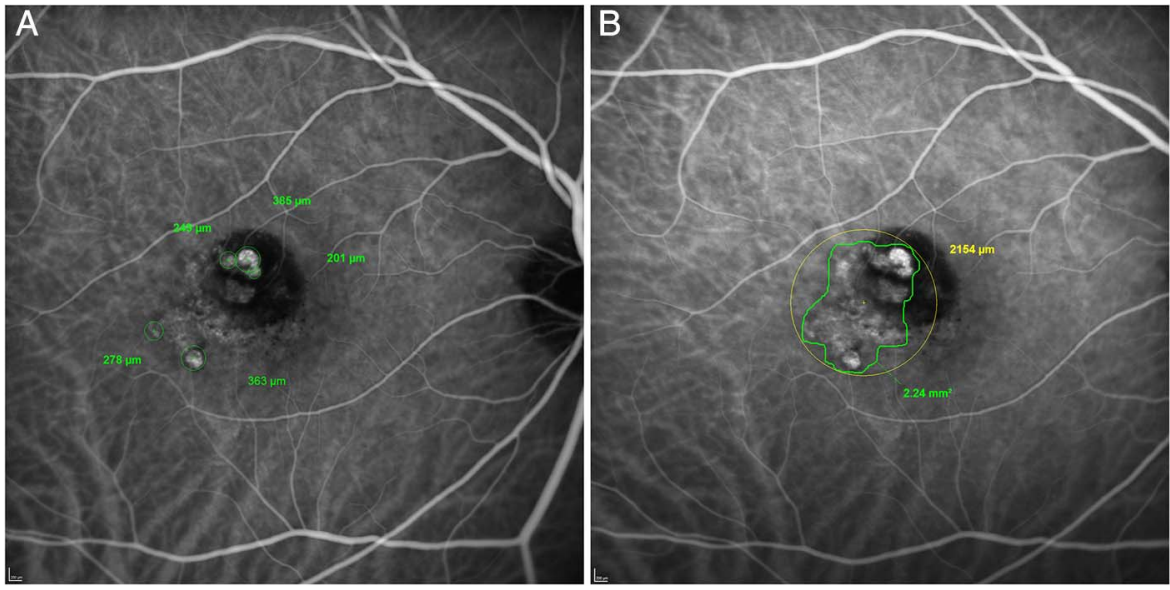

used to describe a typical polyp using ICGA-nodular hyperfluorescence on stereoscopic ICGA (91.8\%), hypofluorescent halo around the nodule (68.9\%) and pulsation during dynamic ICGA (6.6\%). We believe that stereo-pair ICGA images are essential in the diagnosis of PCV, as it allows the determination of nodularity (the most common diagnostic feature) and the depth of the lesions, which helps to differentiate vascular polyps from retinal lesions such as macroaneurysms, large microaneurysms and retinal angiomatous proliferation. This was of particular importance because spectral domain optical coherence tomography (SD-OCT) was not widely available in 2008 when this trial was underway. In future studies, SD-OCT may play an important role in determining the depth and shape of the PCV lesion.

Dynamic ICGA is an essential component of PCV diagnosis, as it allows identification of two of the six diagnostic criteria. The exact boundaries of abnormal vascular channels of the PCV lesion are best visualised in the early phase of the angiogram (within $30 \mathrm{~s}$ ) and may not be seen as clearly after 1 min due to complete filling of the normal choroidal vessels. In addition, the presence of pulsation cannot be detected without the use of dynamic ICGA.

Table 1 Characteristics of polypoidal choroidal vasculopathy (PCV) lesion based on grading of indocyanine green angiography

\begin{tabular}{|c|c|c|c|}
\hline & $\begin{array}{l}\text { Mean } \\
( \pm S D)\end{array}$ & Minimum & Maximum \\
\hline \multicolumn{4}{|l|}{ Polyps } \\
\hline Number of polyps & $4.1(2.0)$ & 1 & 9 \\
\hline $\begin{array}{l}\text { Diameter of smallest polyp within a } \\
\text { single eye }(\mathrm{mm})\end{array}$ & $0.19(0.1)$ & 0.1 & 0.4 \\
\hline $\begin{array}{l}\text { Diameter of largest polyp within a } \\
\text { single eye ( } \mathrm{mm})\end{array}$ & $0.35(0.2)$ & 0.15 & 1.45 \\
\hline Total polyp area $\left(\mathrm{mm}^{2}\right)$ & $0.25(2.0)$ & 0.025 & 2.1 \\
\hline \multicolumn{4}{|l|}{ BVN } \\
\hline BVN area $\left(\mathrm{mm}^{2}\right)$ & $2.87(2.5)$ & 0.07 & 10.52 \\
\hline $\begin{array}{l}\text { Percentage of total area accounted } \\
\text { for by BVN (\%) }\end{array}$ & $85.5(15.4)$ & 28.0 & 98.8 \\
\hline \multicolumn{4}{|l|}{ Total PCV lesion } \\
\hline Total lesion area $\left(\mathrm{mm}^{2}\right)$ & $3.1(2.6)$ & 0.2 & 10.7 \\
\hline Greatest linear diameter (mm) & $2.5(1.2)$ & 0.7 & 5.1 \\
\hline
\end{tabular}

The abnormal vascular channels began to fill within 7.3-32 s from injection. Therefore, the use of dynamic ICGA for the first $30 \mathrm{~s}$ is sufficient to detect these vessels. In all cases, the polyps started to fill within $40.4 \mathrm{~s}$ from injection, with a mean of $21.9 \mathrm{~s}$, which corresponds to the original description of early, intense focal hyperfluorescence on ICGA. ${ }^{12}$ Therefore, the use of either the 1-min or 3-min stereo-pair ICGA images should be adequate to assess the presence, location and extent of polyps and a later stereo-pair at $\geq 10$ min may not be necessary. True late-onset focal hyperfluorescences (appearing after $6 \mathrm{~min}$ ) are more likely to be focal ICG staining of unhealthy RPE or choroidal vascular knuckle showing through focal RPE atrophy (window defect). In both cases, stereo viewing would help to differentiate them.

In this series, 33 of 61 cases $(54.1 \%)$ presented with subretinal haemorrhage. This highlights the importance of haemorrhage as diagnostic criteria for PCV, as has previously been described. ${ }^{13}{ }^{16}{ }^{19-23}$ Ahuja et al $^{17}$ reported that $85 \%$ of patients with haemorrhagic and exudative detachments had evidence of PCV on ICGA.

In several earlier papers, the presence of orange subretinal

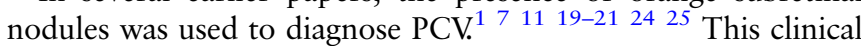
feature was seen in $55.7 \%$ of cases in this study, thus highlighting that not all cases of PCV manifest with characteristic nodules and the need for ICGA to identify the PCV lesion.

The strengths of this study include the use of standardised imaging protocols and equipment on all cases of PCV. The diagnosis of PCV was made by applying stringent diagnostic criteria by expert graders from the Central Reading Center. The main limitation is the lack of data from OCT, which can potentially be used as one of the diagnostic criteria. In future studies, SD-OCT can play an invaluable role in identifying features which are characteristic of PCV, and confirming the location of the hyperfluorescent nodule.

In conclusion, we have described the standardised imaging protocols, grading methods and diagnostic criteria used to diagnose PCV and highlighted important features in a prospective series of patients with PCV. It is hoped that these detailed techniques, and the baseline characteristics described, can be useful to clinical practice and future randomised controlled trials on PCV.

Collaborators Members of the EVEREST Study Group: (1) Adrian Koh, MD FRCS, National Healthcare Group Eye Institute, Tan Tock Seng Hospital, Singapore; (2) Won Ki Lee, MD PhD, Seoul St Mary's Hospital, The Catholic University of Korea, Seoul, Korea; (3) Lee-Jen Chen, MD, Mackay Memorial Hospital, Taipei, Taiwan; (4) Shih-Jen Chen, MD PhD, Taipei Veterans General Hospital, Taipei, Taiwan; School of 
Medicine, National Yang-Ming University, Taipei, Taiwan; (5) Hak Young Kim, MD PhD, Kangnam Sacred Heart Hospital, Hallym University, Seoul, Korea; (6) Timothy Y Lai, MD FRCS, Department of Ophthalmology and Visual Sciences, The Chinese University of Hong Kong, Hong Kong, China; (7) Paisan Ruamviboonsuk, MD, Rajavithi Hospital, Bangkok, Thailand; (8) Tock H Lim, FRCSEd, National Healthcare Group Eye Institute, Tan Tock Seng Hospital, Singapore.

Contributors CST and THL were involved in the conception and design of the study. CST, WKN and THL were involved in the analysis, interpretation and drafting of the manuscript. CT, WKN, JPC, NWT and THL were involved in the finalisation of the manuscript.

Funding This work was supported by Novartis Pharma AG, Basel, Switzerland grant number NCT00674323 (clinicaltrials.gov).

Competing interests CST receives travel support from Bayer, Heidelberg Engineering and Novartis. He also receives research support from the National Healthcare Group Clinician Scientist Career Scheme Grant (CSCS/12005). WKN and JPC have no competing interests in the subject of this paper. NWT receives travel support from Novartis. THL receives travel support from Novartis, Bayer and Heidelberg Engineering.

Patient consent Obtained.

Ethics approval NHG Domain Specific Review Board (DSRB).

Provenance and peer review Not commissioned; externally peer reviewed.

Open Access This is an Open Access article distributed in accordance with the Creative Commons Attribution Non Commercial (CC BY-NC 4.0) license, which permits others to distribute, remix, adapt, build upon this work non-commercially, and license their derivative works on different terms, provided the original work is properly cited and the use is non-commercial. See: http://creativecommons.org/ licenses/by-nc/4.0/

\section{REFERENCES}

1 Yannuzzi LA, Sorenson J, Spaide RF, et al. Idiopathic polypoidal choroidal vasculopathy (IPCV). Retina 1990;10:1-8.

2 Stern RM, Zakov ZN, Zegarra $\mathrm{H}$, et al. Multiple recurrent serosanguineous retinal pigment epithelial detachments in black women. Am J Ophthalmol 1985;100:560-9.

3 Imamura Y, Engelbert M, lida T, et al. Polypoidal choroidal vasculopathy: a review. Surv Ophthalmol 2010;55:501-15.

4 Lim TH, Laude A, Tan CS. Polypoidal choroidal vasculopathy: an angiographic discussion. Eye (Lond) 2010;24:483-90

5 Byeon SH, Lee SC, Oh HS, et al. Incidence and clinical patterns of polypoidal choroidal vasculopathy in Korean patients. Jpn J Ophthalmol 2008;52:57-62.

6 Lafaut BA, Leys AM, Snyers B, et al. Polypoidal choroidal vasculopathy in Caucasians. Graefes Arch Clin Exp Ophthalmol 2000;238:752-9.

7 Tan CS, Ngo WK, Lim LW, et al. A novel classification of the vascular patterns of polypoidal choroidal vasculopathy and its relation to clinical outcomes. Br J Ophthalmol 2014;98:1528-33.
8 Gomi F, Tano Y. Polypoidal choroidal vasculopathy and treatments. Curr Opin Ophthalmol 2008;19:208-12.

9 Cho M, Barbazetto IA, Freund KB. Refractory neovascular age-related macular degeneration secondary to polypoidal choroidal vasculopathy. Am J Ophthalmol 2009;148:70-8.

10 Koh A, Lee WK, Chen LJ, et al. EVEREST study: efficacy and safety of verteporfin photodynamic therapy in combination with ranibizumab or alone versus ranibizumab monotherapy in patients with symptomatic macular polypoidal choroidal vasculopathy. Retina 2012;32:1453-64.

11 Spaide RF, Yannuzzi LA, Slakter JS, et al. Indocyanine green videoangiography of idiopathic polypoidal choroidal vasculopathy. Retina 1995;15:100-10.

12 Yannuzzi LA, Wong DW, Sforzolini BS, et al. Polypoidal choroidal vasculopathy and neovascularized age-related macular degeneration. Arch Ophthalmol 1999;117:1503-10.

13 Uyama M, Wada M, Nagai Y, et al. Polypoidal choroidal vasculopathy: natural history. Am J Ophthalmol 2002;133:639-48.

14 Kwok AK, Lai TY, Chan CW, et al. Polypoidal choroidal vasculopathy in Chinese patients. Br J Ophthalmol 2002;86:892-7.

15 Scassellati-Sforzolini B, Mariotti C, Bryan R, et al. Polypoidal choroidal vasculopathy in Italy. Retina 2001;21:121-5.

16 Sho K, Takahashi K, Yamada H, et al. Polypoidal choroidal vasculopathy: incidence, demographic features, and clinical characteristics. Arch Ophthalmol 2003;121:1392-6.

17 Ahuja RM, Stanga PE, Vingerling JR, et al. Polypoidal choroidal vasculopathy in exudative and haemorrhagic pigment epithelial detachments. Br J Ophthalmol 2000;84:479-84.

18 Gomi F, Ohji M, Sayanagi K, et al. One-year outcomes of photodynamic therapy in age-related macular degeneration and polypoidal choroidal vasculopathy in Japanese patients. Ophthalmology 2008;115:141-6.

19 Maruko I, lida T, Saito M, et al. Clinical characteristics of exudative age-related macular degeneration in Japanese patients. Am J Ophthalmol 2007;144:15-22.

20 Lee MW, Yeo I, Wong D, et al. Photodynamic therapy with verteporfin for polypoidal choroidal vasculopathy. Eye (Lond) 2009;23:1417-22.

21 Wen F, Chen C, Wu D, et al. Polypoidal choroidal vasculopathy in elderly Chinese patients. Graefes Arch Clin Exp Ophthalmol 2004;242:625-9.

22 Byeon SH, Lew YJ, Lee SC, et al. Clinical features and follow-up results of pulsating polypoidal choroidal vasculopathy treated with photodynamic therapy. Acta Ophthalmol 2010;88:660-8.

23 Tan CS, Wong HT, Lim BA, et al. Polypoidal choroidal vasculopathy causing massive suprachoroidal haemorrhage. Eye (Lond) 2007;21:132-3.

24 Wakabayashi T, Gomi F, Sawa M, et al. Marked vascular changes of polypoidal choroidal vasculopathy after photodynamic therapy. $\mathrm{Br} J$ Ophthalmol 2008;92:936-40.

25 Okubo A, Hirakawa M, Ito $\mathrm{M}$, et al. Clinical features of early and late stage polypoidal choroidal vasculopathy characterized by lesion size and disease duration. Graefes Arch Clin Exp Ophthalmol 2008;246:491-9. 